\title{
Usability in a Cultural Context: A Report on the Scope, Process and Research Results of CultUsab - The Cultural Usability Project*
}

\author{
Torkil Clemmensen ${ }^{1}$, Pradeep Yammiyavar ${ }^{2}$, Rikke Ørngreen ${ }^{3}$, and Dinesh Katre ${ }^{4}$ \\ ${ }^{1}$ Department of Informatics, Copenhagen Business School, Denmark \\ tc.inf@cbs. dk \\ ${ }^{2}$ Indian Institute of Technology Guwahati, India \\ pradeep@iitg.ernet.in \\ ${ }^{3}$ Danish School of Education, Aarhus University, Denmark \\ rior@dpu.dk \\ ${ }^{4}$ Human-Centred Design \& Computing, C-DAC, Pune, India \\ dinesh@cdac.in
}

\begin{abstract}
This paper focuses on presenting and discussing the aim, context, challenges, results, and impact of the Cultural usability project named as CultUsab. This project was a four year international research effort from 2006 to 2009 , which was supported by a grant from the Danish Research Councils for Independent Research in Culture and Communication. The project aimed at innovating processes in Information and Communication Technology development through an understanding of culturally sensitive aspects of usability evaluation methods ${ }^{1}$.
\end{abstract}

Keywords: Culture, Usability, Evaluation techniques, cross-cultural testing.

\section{Introduction}

With the advent of globalisation and the information technology revolution in developing countries, it is not anymore possible to overlook the aspect of culture in the design of user interfaces and interactive products. There is a need to understand and accept that there are significant differences in how people with different cultural backgrounds respond to directions and test methodologies. From the laboratory of the very large IT companies in Beijing to the design departments at India's finest institutions of higher education, there is a call for adequate methods and techniques for designing human-computer interaction. Usability issues such as how to support input to computers of the many Chinese characters within a classic windows and mouse paradigm, or how in a multilingual and multicultural country as India - to design and evaluate the usability of interfaces to Automatic Teller Machines and other textbased interactive products, must be addressed. Denmark and Europe face complex

\footnotetext{
${ }^{*}$ See Appendix A for a list of participants in the CultUsab project.

${ }^{1}$ See www.culturalusability.com 
challenges to the quality of information and communication technology, as these societies turn increasingly multicultural and must provide networked information to both ethnic majorities and minorities. Despite of these challenges, there is not any kind of formal methods which guides the evaluation of a product to a certain standard while keeping sensitivity to cultural issues.

The CultUsab project therefore took, as a point of departure, the issues of how to avoid cultural bias in requirements elicitation and usability data collection. Which user-based evaluation methods do address cultural diversity in both the moderator and user during a testing session? The project studied fundamental and widespread assumption that the usability evaluator requires to have the same cultural background as that of the test user in order to completely understand how users will respond to the test instructions and testing methodology. Furthermore, the project aimed to understand effective ways to obtain test users' usability feedback without actually disguising the usability problems. The project's research question was:

What is the impact of culture on the results of established methods of usability testing?

Hereunder, investigating:

- How are the different components of a usability test, e.g. planning, performing and reporting, influenced by a cultural diversity of users and contexts of use?

- How are cultural backgrounds taken into account when recruiting and describing usability test users?

- Which form of relations and communications between evaluators and test users are most effective in terms of finding relevant usability problems in culturally localized applications?

- What is the nature of common cross culturally related usability problems, and what is a good quality of cultural usability of information and communication technology?

The CultUsab project aimed to investigate the impact of culture on the results of established methods of usability testing. The production and use of technologically advanced information and communication applications are no longer restricted to the western hemisphere and there are indications that usability testing procedures developed for use in Europe or the US do not necessarily give reliable results in countries of the eastern hemisphere such as India, China or Malaysia. The project was an indepth investigation of the cultural specifics that go into usability test situations in three countries: Denmark, India and China. In a second phase we explored possible developments of the testing methods in order to avoid cultural bias and produce comparable results across countries of the world. The project was a collaborative one, involving senior researchers in all three countries where testing occur, as well as advisers from worldwide companies based in US and Europe and from institutions elsewhere.

In this paper, we present the research background and theoretical foundation of the project, followed by methodological issues (research design and approach) including a process portrayal of the partners' collaboration between and across cultures. The achievements from the cultural usability project are described thereafter, ending with a discussion about its impact. 


\section{Research Background - Usability Evaluation Methods}

A focus in the CultUsab project was Usability Evaluation Methods (UEMs), as defined by $[12]^{2}$. In the industry, a wealth of UEMs is used to evaluate computer software user interfaces and other interactive products: Inspection methods, Workplace observation, Think-Aloud Usability Test, etc. Both in the industry and in research there is an interest in understanding cultural issues because there are numerous cultural factors that influence usability evaluation results. Some have to do with culturally biased guidelines and procedures in using a specific UEM, while others are related to other types of cultural differences appearing in test situations. There is an entire spectrum of factors ranging from those completely independent of the UEM to those that are practically built-in, in a particular UEM. For reasons of comparability, the CultUsab project had to consider more than one type of UEM.

The theoretical part of the project analyzed the concept of 'cultural usability' through analysis of the use of UEMs within a cultural and social diversity of users and contexts. The international diversity of users and contexts of use was an expansion of the traditional usability research, which at the time of the project start was based on more simple, regionally specific conceptions of users [4, 17]. The research methodology, mostly qualitative, was designed to allow for in depth investigation of the conceptual and practical layers of user and context representations in established UEMs.

The discussion about culturally localised interfaces has been fairly conclusive on the point that localization is not just mere translation of text, it's more than that [22, 24]. To locally adapt user interfaces, we must use usability engineering methods similar to those used in the development of original user interface. However, the existing practice derived from the west of migrating software from a source culture to a target culture, may work in the design and implementation phase but not in the usability evaluation phase [41]. For example, in Malaysia having a test user of higher rank than the experimenter will result in more negative comments about the product than having a test user of lower rank than the evaluator. In some countries testing subjects individually should be avoided, as little information may be retrieved [13]. In an interview study done in India, those participants with a similar socio-cultural background as the interviewer (India) brought more usability problems than participants who were interviewed by the interviewer with a different socio-cultural background (in this case Anglo-American) [35]. Others have raised similar issues: Do language and cultural differences between staff and participants negate the outcome of usability tests? Are foreign nationals good representatives of users in their home country? These practical issues are of great importance to the design and use of usability evaluation methods.

The background for many studies of cultural aspects of usability were at the time of the beginning of the CultUsab project [15]'s cultural dimensions: power distance, individualism-collectivism, masculinity-feminism and uncertainty avoidance. Most culture and design theorists, many professional designers across all disciplines and also some users believed that these cultural dimensions pervade every human activity and every artifact, including user interfaces [21]. However, opponents of this

\footnotetext{
${ }^{2}$ UEMs is a broad term for analytical and empirical methods that the usability professional use to evaluate the interaction of the human with the computer; The purpose being to identify aspects of this interaction that need to be improved to increase the usability of the product.
} 
approach argued that the current process for the design of universally usable systems was not appropriate, because of its overdependence on guidelines, difficulty of determining the user from the present cultural grounds, its tendency to build stereotypes which later become design rules, and its treatment of different cultures with one specific language that doesn't take into account cultural heterogeneity. Instead, these researchers saw culturally determined usability problems in interfaces as caused by the users' (mis-)understandings of the representations whose meaning lie in the culture-specific context [1]. Their conceptualization of cultural usability looked more like social psychological approaches to culture that include the establishment of 'social facts' and peoples' sense of the 'reality' of social groups, and see these as effects of peoples' use of symbols to construct their social reality; processes that again are firmly related to culture and communication [19].

The CultUsab project was designed to see these processes as important for cultural usability. For example, in pilot studies in India and Denmark of the thinking aloud usability test method [5, 37], the test users quickly realized that some test evaluators did not belong to the user's own social group, and acted accordingly by explaining to the foreign evaluator aspects of the test application that would seem to be obvious and not require explanation to an evaluator from the same group. In the end, this meant that some relevant usability problems were not identified due to cross cultural issues. The cultural usability project used these findings to constructively critique and extend the existing Usability Evaluation Methods.

\section{Research Approach and Design}

The CultUsab project was based on a moderate universalism in the sense of [26]: One - Perhaps there indeed are cross cultural universal usability factors or there are not necessitating empirical documentation. Two - Universal usability will most probably be found on the level of theoretical principles rather than on the level of observed phenomena. Three - we need to make assumptions about universal usability to help organize data into general theories. With this as the base, the project looked away from the two sisters of universalism [32]: evolutionism (one society is more advanced than others) and relativism (societies must be understood from their own perspective) approaches, in order to create the best ground for comparability of results and collaboration among the researchers in the project.

\subsection{Social Psychological Approach to Cultural Usability}

The CultUsab project intended to apply a social-cognitive model of culture [16] that conceptualized culture as a loose network of domain-specific cognitive structures (including theories, beliefs), and, furthermore, argued that an individual can hold more than one cultural meaning system, even if the systems contain conflicting cultural theories. The theory says that depending on the accessibility, availability and applicability of such cultural knowledge, cross-cultural differences may impact usability.

Accessible cultural knowledge is approached as meaning systems that are widely shared among members of a cultural group and frequently used in communication 
among members and thus becomes chronically accessible. In a usability test situation, where people under time pressure look for readily available and widely accepted solutions to a problem, the chronically accessible knowledge will be used and typical cultural group differences will emerge.

It is however not sufficient to have task conditions that favor the use of chronically accessible cultural knowledge. Since individuals in a society increasingly are polycultural in their background and thus have more than one implicit theory of how to perceive and act in a given situation, the individual choose or implicitly apply the theory that is most accessible in that situation. Therefore, in the study of UEMs it is sometimes necessary to ensure the availability of culturally accessible knowledge by including ways to activate or 'prime' this knowledge. Such primers can be cultural icons and pictures. For example, we will test localized IT applications that contain culturally specific icons and pictures that can prime evaluators' and test users' culturally specific knowledge systems, while they complete a behavioral strategy such as a think aloud usability test.

The CultUsab project dealt with the assumption about appropriateness of applying cultural knowledge by pairing evaluator and users of different respectively similar socio-cultural backgrounds. In order not to miss significant parts of the social realities of a postmodern world [2], the project studied the performance of UEMs at different 'home grounds' in China, India and Denmark. The aim was to do a great variety of sub-studies in order to estimate the universality of claims about cultural usability in the project. The glue that bound the sub-studies together was that individual researchers were present at the studies and field experiments that were done at the other researchers' home grounds.

\subsection{Activities and Methods}

The project had an exploration phase and a test phase. The exploration phase summarized the findings from pilot studies from Denmark, India, and China, and did a literature review of cultural usability. The aim was to create a common basis among partners for the study of the concept of Cultural Usability and for using ethnographic research methods; this was followed by ethnographic studies in China, India and Denmark to provide answers to the explorative parts of the research question and an international seminar to focus the analysis of data and the creation of hypotheses about cultural usability. The test phase formulated precise hypotheses about methods to test cultural usability. Field experiments were done in all three countries to find answers to the test part of the research question. At the end of the project, an international working conference was held to distribute the results and discuss cultural usability with researchers and practitioners in all three countries. Some of the papers from this conference are published in this book on Human Work Interaction Design with the theme of "Social, cultural and organizational aspect of usability".

\subsection{Challenges Involved in Cultural Usability Research Collaboration}

Hereunder we provide insight into the work process of the project, not as a research design, but as it took place, between the project partners. This process view provides insight into the explorative methodology of opening and understanding the UEM 
applied in the project and serves as a basis for understanding the findings in the following section.

While the Cultusab project itself originated around the idea of understanding cross cultural issues in usability testing, the deliberations and exchanges between the involved researchers themselves would have become a subject of 'experiment' to any other researcher in cross cultural issues. It has been a rich cauldron of experiences for all its team members in more ways than one.

There were the usual time zone differences, administrative procedural differences, time consuming official regulations of individual institutions, confusion regarding fluctuations in international exchange value of the sponsors currency etc, but what is more interesting to the researchers in HCI and related fields is the differences in mental models each team member brought to the discussions to understand and contribute to the project and the theories applied. Each member's mental model contributed positively to the outcome however different they seemed to be. In terms of relative weights, it is difficult to say to which extent partners were driven by individual attitude, professional discipline and place of origin while using their mental models. Differences if any were attributable to academic points of view that arise in any research work.

What got revealed as an outcome of interaction in between the project team members is as follows:

a. While there are commonalities between two geographically adjacent populations (example: China and India) there were indeed differences in understandings and interpreting such proximate cultures. On the other hand, geographical distances between two cultures (example: Europe and India) need not increase the differences nor reduce the commonalities. While cultural practices mattered; the question that remained was at which points in the usability design methodology should culture be considered as an influencing factor.

b. Though populations (example: India) apparently seem to have a mono culture (as seen by a non-Indian), on close examination there are multitude cultures within the culture, some sort of multiple variations of the same culture. This implied that results of a test conducted in one region in a country as a large as India need not necessarily be valid all over India. However, there was indeed an underlying common cultural essence in India. This may pertain to similar findings, of conducting tests in two very different organizational cultures. Consequently, trying to identify common behavioral practices say in the use of mobile phones would be a Herculean task for a Usability researcher or tester in cultures like India and China.

c. Some team partners at first attempted to apply known culture theories such as Hofstede cultural dimensions to explain the results of the tests or provide a convincing framework to build upon heuristics for Usability testing. There were, however, academic arguments within the team that recommended the examination of other theories such a Nisbett's culture theory as framework. It seems that neither Hofstede nor Nisbett, even after both being utilized on the same data, are able to help in predicting user responses and behavior in 
multi cultures like India. This points out for a real need of a more contemporary theory of culture that can be specifically useful to cross cultural usability researchers. Several delegates in the conferences of the CultUsab project and the HWID events also echoed the same lack of robust cultural theories and frame works for the use of HCI and usability researchers.

d. The team deliberately avoided on agreeing to a common definition of 'culture' as it was felt that the suggestion by a member of the team to work on the basis of 'commonalities' in usability rather than the 'differences' was more appropriate. A broad definition of culture would suffice for the team which was more interested to make inroads into HCI and Usability across cultures. Indeed the lessons seem that it is only when differences are the focus that precise definition of culture needs to be agreed upon before work commences involving such large cross cultural international research teams working across different countries.

Organizational constrains are expected in such international collaborating teams. The team discovered early in the process that it is not really easy to use one of the new technologies such as video and voice over IP conferencing, instant messaging etc. Very quickly members from different global locations did agree that there were neither cultural differences nor defiance regarding limitations of virtual meetings! Rather the problem occurred due to human factors such as time shift, non availability, band width failure, etc., and it seemed the more reliable format of email technology indigenously operated by each of the team member, served the purpose better. Also, a project website helped communicating with members, who followed the project on the sideline.

One of the difficult situations was regarding the status of research scholars working in such projects. Adherence to maintaining the difference in Teacher - Student status in India and China are necessary due to cultural traditions and prevalent practices in these countries; whereas the student-teacher relationship is almost at the equal level in other cultures. Students in India and China are relatively of younger age at the postgraduate level and are required to only communicate internationally with project teams through the teacher. Academic freedom given to students in India and China is supervised by a teacher; whereas else where it may not be so. It did take some time to adjust to such cultural practices. Nonetheless, research students who went on exchange visits and attended conferences as part of the CultUsab project learnt immensely. The CultUsab project's investments on these research scholars by planting the 'seeds of interest' in Cultural usability research can easily turn out to be one of the most valuable return of investment in terms of the future. The need to have local team members present during data collection and experimentation by a team not belonging to that place was reinforced during the course of this project.

The project was a great cross cultural experience both personally as well as academically for all its participating members.

\section{Major Achievements of the CultUsab Project}

This section present the important publications, research problems, techniques, methods, solutions and network of international researchers that emerged out of the 
CultUsab project. This is done through answering each of the sub research questions of the project (that were presented in the first section of the paper).

\section{How are the different components of a usability test, e.g. planning, performing and reporting, influenced by a cultural diversity of users and contexts of use?}

The CultUsab project studied the impact of culture on the results of established methods of usability testing. First, an initial literature study found that culture impacts all the different components of a usability test [7] (see Figure 1). The conclusion pointed to the importance of matching the task presentation to users' cultural background, the different effects of thinking aloud on task performance between Easterners and Westerners, the differences in nonverbal behaviour that affect usability problem detection, and, finally, the complexity of the overall relationship between user and evaluator when they have different cultural backgrounds.

This was supported by field studies of Think Aloud usability tests in companies in Copenhagen, Beijing, Mumbai [9, 10, 29] that indicated culturally variant influences on the test moderator's focus on client satisfaction, task performance and test users' comfort; formal vs. informal nature of evaluation interviews; evaluation after each sub-task vs. evaluation after the whole task; and culturally specific understandings and practices concerning what essentially are part of a usability test. The field studies found a number of country-specific and also a number of cross cultural context variables for usability testing, see table 1 .

Nielsen and Chavan [25] in their field studies of remote usability testing found that compared to a task list presentation, a scenario based description of the computing task given to low power status participants made them more innovative in finding and suggesting solutions to usability problems.

Plocher et al. [23, 27, 28] studied participants' familiarity with the evaluated software's information architecture, and found that this vary between cultural groups, because of cultural differences in structures of categories.

\section{How are cultural backgrounds taken into account when recruiting and describing usability test users?}

The second finding from the project was that culture influences the ways of involving participants in usability evaluation. Joshi [18], in a review of typical real-life projects from a variety of companies in the Indian IT industry, found that Usability professionals in India come in late in design, they are often replaced by software engineers, their user centered design focus is not easily accepted in large software engineering companies), and their human-oriented, holistic approach to interaction design does not go well with the preferred step-by-step, atomistic, approach of software engineering methods. However, more research is needed in this area.

\footnotetext{
Which form of relations and communications between evaluators and test users are most effective in terms of finding relevant usability problems in culturally localized applications?
} 


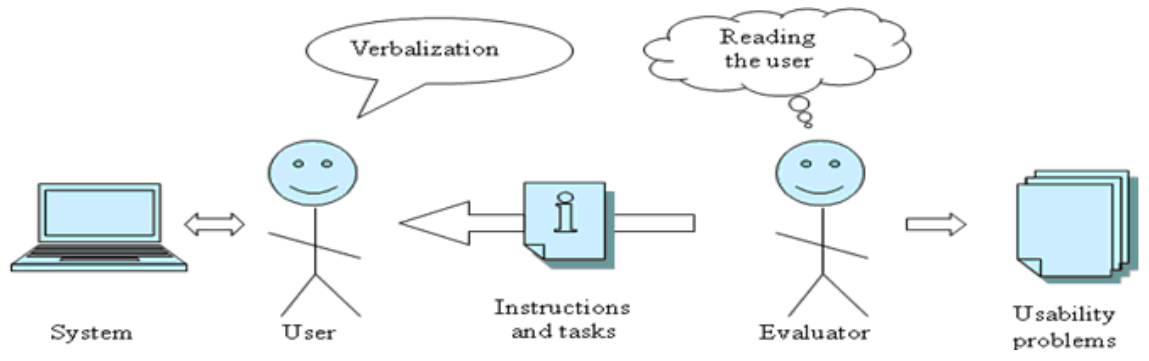

Fig. 1. Reference model of usability testing

Table 1. The paradigmatic context-factors in a cross cultural a usability test. Note: 'Yes' means that the part of the usability test is influenced by the context-factor, 'No' means that it is not influenced by the context-factor.

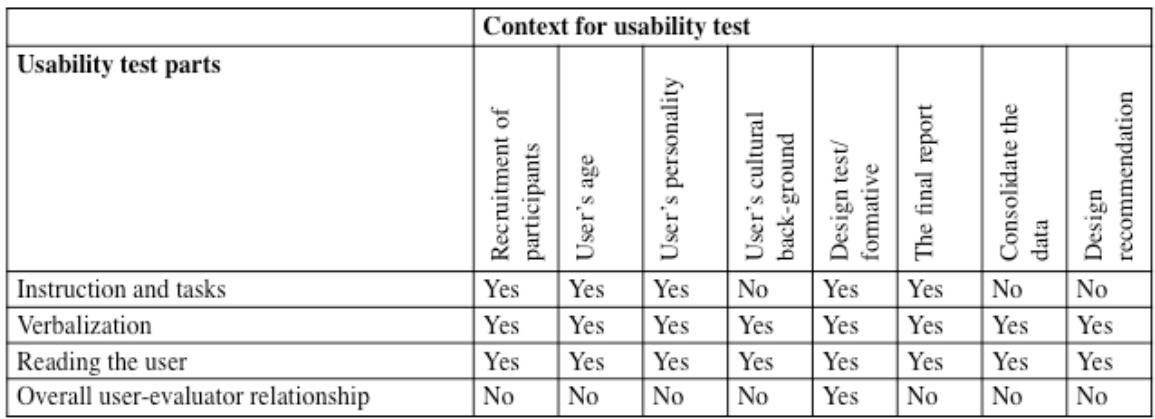

The third finding from the project was that cultural background determines what is effective communication in terms of finding relevant usability problems [6, 31]. Moderators with background similar to that of the test users gave better descriptions, and found more frequently the most severe, important problem with the software tested. In the interview-style parts of the tests, local moderators facilitated more explanations and descriptions from users, while there were no differences in the process when the moderators acted as passive observers in line with classic think aloud. Research in the relationship and communication patterns in usability tests is on its way in a PhD study [30], already indicating that an important factor in cross cultural usability testing is the relationship management. Sun and Shi [34] studied the effect of language in usability testing in China and found that speaking Chinese made the moderator more assisting in detail, and encouraging users more frequently; while when speaking English, the moderator had to pay more attention to the screen to understand what was going on.

Yammiyavar et al [38-40] observed that the use of Non-verbal Cues in Usability Evaluation seemed to be an important corollary to the users verbal statements in detecting usability problems; they found first that the Indian users found the Think aloud exhausting, interfering with their tasks, and second that gestures were interpreted in 
Table 2. The spread of user behaviours over the time duration of a usability test. The figure is from [39].

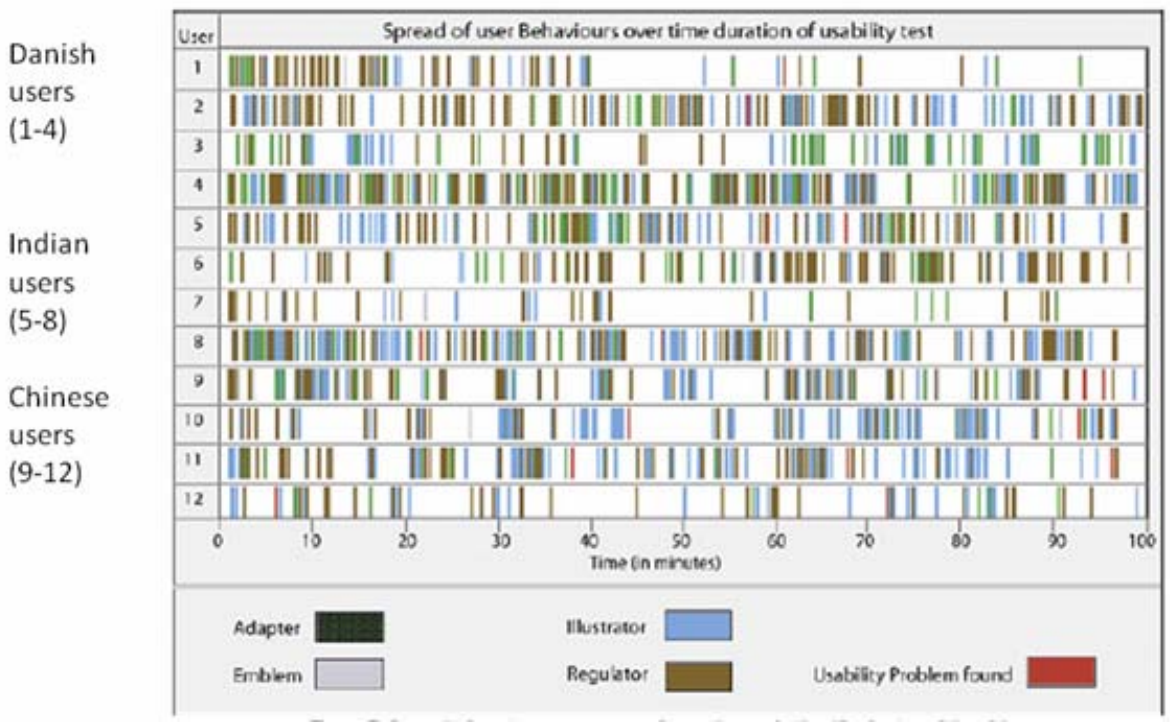

the same way by user and moderators when they were familiar, but not when the moderator was a stranger. Surprisingly, there was very small overall difference in the use of non-verbal gestures among the users form different countries, see Table 2. Kumar et al [20] tested in India a method for retrospective usability evaluation that previously had helped Danish users overcome their difficulties with thinking aloud, and found it to be sensitive to information on users' motives and intentions in different cultural contexts, which may be helpful when comparing usability problem lists in cross cultural usability evaluations.

\section{What is the nature of common cross culturally related usability problems, and what is a good quality of cultural usability of information and communication technology?}

The fourth finding from the CultUsab project was that the nature of common cross culturally related usability problems, and what is a good quality of cultural usability of information and communication technology; vary across country and stakeholder groups. Hertzum et al [14] did repertory-grid interviews in three countries (China, Denmark, India). They investigated how stakeholders (developers, users) personal constructs of the use of their own software systems were affected by their cultural background. The found that find that for the user group frustrating and useful systems are experienced similarly, whereas for the developers frustrating systems are experienced similarly to easy-to-use systems. Looking at the most characteristic construct for each participant they find that Chinese participants use constructs related to security, task types, training, and system issues, whereas Danish and to some extent Indian participants make more use of constructs traditionally associated with usability (e.g., easy-to-use, intuitive, and liked). 


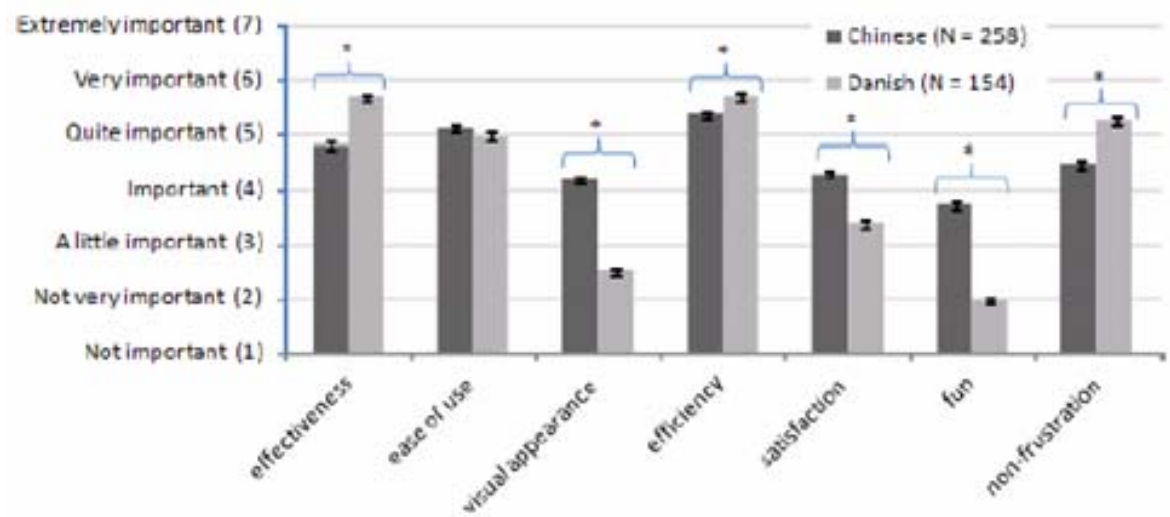

Fig. 2. Average responses to seven scales of usability. the scales marked with an asterisk show a significant difference between Chinese and Danish respondents. Error bars shows the standard error of mean. The figure is adapted from Frandsen-Thorlacius et al (2009).

In a questionnaire study in Denmark and China, Frandsen-Thorlacius et al. [11] found that the notion of usability, its aspects, and their interrelations are not always constant across cultures, see figure 2.

\section{Conclusion on Findings from the CultUsab Project}

\subsection{Practical Application of Results}

The project was designed to have significant societal impact on issues related to cultural aspects of interaction design and usability testing. Danish Usability professionals need to improve their understanding of usability in other parts of the world and their ability to configure usability evaluation methods cross culturally in e.g. Indian and Chinese settings or ethnic minority settings within Europe and Denmark. An understanding of the cultural aspects of usability was meant to help the designer and developers to analyze the ontology of the application domain of a system by revealing the semantics of the domain from the users' many points of view. The openness of the technology for a wide range of interpretations makes it very important to develop UEMs that help the designers and developers to investigate the use of technology on many levels of detail within society. This was seen as very important in efforts to coordinate between incompatible system developments methods such as the natural science-oriented object-oriented analysis and design approach versus the humanitiesoriented interaction design approach to usability [8].

\subsection{Publication of Results}

Results of the project were published in high level international HCI journals including Interacting with Computers. Publications included psychology outlets such as the International Ergonomics Conference. The current book was one of the target outcomes of the HWID - CultUsab conference held at the end of the project in the month 
of October 2009 in Pune, India. The CultUsab project was presented and discussed with researchers and industry at appropriate conferences including the annual Danish HCI research symposium, the INDIA HCI conferences and HCI International 2007, Beijing, the American CHI conference, and the International ICIS Information Systems conference. A CultUsab publication received a best paper award at HCI International 2009 and another was nominated for one at CHI2009. A number of workshops were also conducted by the project members travelling across continents, to encourage the participation of students in different institutions.

\subsection{International Collaboration and Methods Development}

In developing the methods of testing intercultural usability evaluation, the project developed and evaluated the methods for doing so. Moreover, the intense collaboration on all levels of this project, from field testing to analysis and publication, strengthened research networks between the countries involved and paved the way for future research in this and related areas to the benefit of all the participating research institutions and researchers and their students. Furthermore, the cooperation with the international research group behind the first Indian Human-Computer Interaction conference,[33], is evident from the paper by Andy Smith (who headed the mentioned research group) published in this book. The project also exploited opportunities to cooperate about research in cultural usability with HCI researchers from the emerging HCI communities in developing countries across the world, e.g. [3, 36].

\subsection{Educational Benefits}

The educational significance of the project lay in the participation of HumanComputer Interaction MSc and $\mathrm{PhD}$ students from Copenhagen Business School, Chinese Academy of Science; B.Des, M.Des and PhD students of Indian Institute of Technology Guwahati. Many students benefited from the global perspectives on human-computer interaction during the workshops. The project was of special interest to students having a multicultural background or expected career in a multicultural environment.

\subsection{Future Research Agenda}

The results of the CultUsab project pointed to the next phase of research in cultural usability. A possible agenda of a future research project is the training of users as part of improving the usability of information and communication technology. As we know from numerous studies, there are high costs associated with learning to use new systems and with the social psychology of the surrounding cultural and communicative processes.

\section{Impact of CultUsab Project}

CultUsab project has been able to attract significant participation of usability researchers and practitioners from various countries and cultures. It has propelled the cultural usability research in both academia and industry. The impact of CultUsab 
project can be observed in terms of its contributions towards advancing the ongoing cultural usability research and setting some new trends.

\subsection{Cultural Usability as an Imperative for Reaching Out to Global Markets}

Cultural usability is gaining serious attention as businesses seek growth through expanding their international markets. Usability companies are now engaged in establishing international networks to offer services to evaluate the cultural usability of products and identify the culture specific demands. Culture specific requirements are now being studied to improve the acceptability of software systems, online business websites and mobile applications.

\subsection{Investigation of Usability Testing Methods to Identify the Cultural Variance}

Usability testing and evaluation methods are now being investigated by researchers to identify the influence of tester's or subject's cultural background in the observation and interpretation of test results. Well known cultural models and theories are being tested, evaluated and extended further to address the diversity of cultures and its dynamically evolving nature. Existing usability research is being re-investigated to find cultural bias.

\subsection{Use of Online Communities and Social Networks}

International social networks and e-mail discussion groups are often being used for reaching out to users with different nationalities. Online surveys are carried out by involving the interested users with different cultural backgrounds. Internet based applications are being developed for remote usability testing particularly for the users in geographically dispersed locations.

\subsection{Internationalization of Design and Usability}

Different models and software frameworks are being explored to allow internationalization of usability for technology products. Here the focus is not only on diversity of languages but also on overall design of product and the diverse design preferences of users belonging to different cultures.

\subsection{Cross-Cultural Partnerships and Collaborations}

Cross-cultural partnerships are being formed by usability practitioners and researchers to carry out cultural usability research. It has boosted imaginative possibilities to collaborate on common ideas with cultural variance.

\subsection{Cultural Usability Research}

Dedicated conferences and workshops are now being organized to focus purely on the theme of cultural usability, internationalization of products and systems. Many $\mathrm{PhD}$. research scholars, students of Masters and Undergraduate level courses are now choosing to focus on different aspects of cultural usability as part of their dissertations 
and academic projects. Outsourcing companies offering information technology services have also started to recognize the importance of addressing the culture specific needs of the foreign clients.

\subsection{Awareness of Cultural Usability}

Through the organization of conferences, workshops, symposiums and seminars, the CultUsab project has been successful in gathering the critical mass of usability researchers, practitioners and students. It has helped in creating a widespread and synergetic workforce of researchers crossing the cultural and national boundaries.

\section{References}

1. Bourges-Waldegg, P., Scrivener, S.: Meaning, the central issue in cross-cultural HCI design. Interacting with Computers 9 (1998)

2. Burawoy, M.: Global Etnography. California Press (2000)

3. Cecilia, M., Baranauskas, C.: HCI in Brazil: Prospect and Challenges. In: INTERACT 2003 - Bringing the Bits together - Ninth IFIP TC13 International Conference on Human Computer Interaction, Zürich, Switzerland, pp. 1081-1083 (2003)

4. Clemmensen, T.: Four approaches to user modelling - a qualitative research interview study of HCI professionals' practice. Interacting With Computers 16(4), 799-829 (2004)

5. Clemmensen, T., Goyal, S.: Cross cultural usability testing. Working paper, Copenhagen Business School, Department of Informatics, HCI research group, 2005-006. 20 (2005)

6. Clemmensen, T., Goyal, S.: Cross cultural usability testing. In: The First All India Human Computer Interaction Conference, Bangalore, India (2005)

7. Clemmensen, T., Hertzum, M., Hornbæk, K., Shi, Q., Yammiyavar, P.: Cultural cognition in usability evaluation. Interacting with Computers 21(3), 212-220 (2009)

8. Clemmensen, T., Nørbjerg, J.: Separation in Theory, Coordination in Practice - Teaching HCI and SE. Software Process - Improvement and Practice 8, 99-110 (2003)

9. Clemmensen, T., Shi, Q.: What is part of a usability test? In: CHI 2008 extended abstracts on Human factors in computing systems, Florence, Italy. ACM, New York (2008)

10. Clemmensen, T., Shi, Q., Kumar, J., Li, H., Sun, X., Yammiyavar, P.: Cultural Usability Tests - How Usability Tests Are Not the Same All over the World. In: Aykin, N. (ed.) HCII 2007. LNCS, vol. 4559, pp. 281-290. Springer, Heidelberg (2007)

11. Frandsen-Thorlacius, O., Hornbæk, K., Hertzum, M., Clemmensen, T.: Non-Universal Usability? A Survey of How Usability Is Understood by Chinese and Danish Users. In: CHI 2009 (2009)

12. Gray, W.D., Salzman, M.C.: Damaged merchandise? A review of experiments that compare usability evaluation methods. Human-Computer Interaction 13(3), 203-261 (1998)

13. Herman, L.: Towards Effective Usability Evaluation in Asia: Cross-cultural differences. In: OZCHI 1996 (1996)

14. Hertzum, M., Clemmensen, T., Hornbæk, K., Kumar, J., Shi, Q., Yammiyavar, P.: Usability Constructs: A Cross-Cultural Study of How Users and Developers Experience Their Use of Information Systems. In: Aykin, N. (ed.) HCII 2007. LNCS, vol. 4559, pp. 317-326. Springer, Heidelberg (2007) 
15. Hofstede, G.: Geert Hofstede's Homepage (2005)

16. Hong, Y.-y., Mallorie, L.M.: A dynamic constructivist approach to culture: Lessons learned from personality psychology. Journal of Research in Personality 38, 59-67 (2004)

17. Isomaki, H., Pohjola, A.: Introducing Multiple Views on Gender and Information Technology. In: Isomaki, H., Pohjola, A. (eds.) Lost and found in virtual reality: Women and Information technology. University of Lapland (2005)

18. Joshi, A.: HCI and SE-The Cultures of the Professions. In: Aykin, N. (ed.) HCII 2007. LNCS, vol. 4559, pp. 108-112. Springer, Heidelberg (2007)

19. Kashima, Y.: Culture, Communication and Entitativity - A Social Psychological Investigation of Social Reality. In: Yzerbyt, V., Judd, C.M., Corneille, O. (eds.) The psychology of group perception - perceived variability, entitativity and essentialism. Psychology Press, NY (2004)

20. Kumar, J., Yammiyavar, P., Nielsen, J.: MindTape technique - a usability evaluation method for tracing cognitive processes in cross cultural settings e-Minds 1(3) (2007)

21. Marcus, A.: Fast forward: Culture class vs. culture clash. Interactions 9(3), 25-28 (2002)

22. Molich, R., Dray, S., Siegel, D.: Tips and Tricks for a Better International Usability Test. In: CHI 2004 Special Interest Group, Vienne, Austria. ACM, New York (2004)

23. Nawaz, A., Plocher, T., Clemmensen, T., Qu, W., Sun, X.: Cultural differences in the structure of categories in Denmark and China. Department of Informatics, CBS, Working Paper nr. 03-2007 (2007)

24. Nielsen, J.: Designing for International Use. In: CHI 1990 (1990)

25. Nielsen, L., Chavan, S.: Differences in Task Descriptions in the Think Aloud Test. In: Aykin, N. (ed.) HCII 2007. LNCS, vol. 4559, pp. 174-180. Springer, Heidelberg (2007)

26. Pepitone, A.: A social psychology perspective on the study of culture: An eye on the road to interdisciplinarianism. Cross-Cultural Research 34(3), 233-249 (2000)

27. Plocher, T., Clemmensen, T.: Comparing User and Software Information Structures for Compatibility. In: Aykin, N. (ed.) HCII 2007. LNCS, vol. 4559, pp. 178-182. Springer, Heidelberg (2007)

28. Qu, W., Sun, X., Nawaz, A., Plocher, T., Clemmensen, T.: Cultural Didderences Between Chinese and Dane in Card Sorting. In: The Eighth Pan-Pacific Conference on Occupational Ergonomics (PPCOE 2007), Bangkok, Thailand (2007)

29. Shi, Q.: A field study of the relationship and communication between Chinese evaluators and users in thinking aloud usability tests. In: NordiChi 2008, Lund (2008)

30. Shi, Q., Clemmensen, T.: Communication pattern and usability problem finding in crosscultural thinking aloud usability testing. In: The Seventh Danish HCI Research Symposium (DHRS 2007), IT University, Copenhagen, Denmark (2007)

31. Shi, Q., Clemmensen, T.: Relationship Model in Cultural Usability Testing. In: Aykin, N. (ed.) HCII 2007. LNCS, vol. 4559, pp. 422-431. Springer, Heidelberg (2007)

32. Shweder, R.A., Bourne, E.J.: Does the Concept of the Person Vary Cross-Culturally? In: Shweder, R.A. (ed.) Thinking Through Culture - Expeditions in cultural psychology. Harvard University Press, London (1991)

33. Smith, A., Dunckley, L., French, T., Minocha, S., Chang, Y.: A process model for developing usable cross-cultural websites. Interacting with Computers 16(1), 63-91 (2004)

34. Sun, X., Shi, Q.: Language issues in cross cultural usability testing: a pilot study in China. In: Aykin, N. (ed.) HCII 2007. LNCS, vol. 4559, pp. 274-283. Springer, Heidelberg (2007)

35. Vatrapu, R.: Culture and International Usability Testing: The effects of Culture in Structured Interviews. Master thesis. Virginia Polytechnic Institute and State University (2001) 
36. Wesson, J., Greunen, D.v.: New Horizons for HCI in South Africa. In: INTERACT 2003 Bringing the Bits together - Ninth IFIP TC13 International Conference on Human Computer Interaction, Zürich, Switzerland, pp. 1091-1095 (2003)

37. Yammiyavar, P., Clemmensen, T., Goyal, S.: Culture as a factor in interaction design testing for interfaces - case study in Indian scenario. In: Humanizing Work and Work Environment - International ergonomics conference, IIT Guwahati, Assam, India (2005)

38. Yammiyavar, P., Clemmensen, T., Kumar, J.: Analyzing Non-verbal Cues in Usability Evaluation Tests. In: Aykin, N. (ed.) HCII 2007. LNCS, vol. 4559, pp. 462-471. Springer, Heidelberg (2007)

39. Yammiyavar, P., Clemmensen, T., Kumar, J.: Influence of cultural background on nonverbal communication in a usability testing situation. International Journal of Design 2(2), 31-40 (2008)

40. Yammiyavar, P., Goel, K.M.: Emphasis on non-verbal cues for interpreting cognitive processes in protocol analysis. In: Indo-Danish HCI Research Symposium, Guwahati, India. The Indian Institute of Technology Guwahati, Guwahati (2006)

41. Yeo, A.: Global-software Development Lifecycle: An Exploratory Study. In: CHI 2001 (2001) 


\section{Appendix A: List of the Main Participants in the CultUsab Project}

Dept. of Informatics, Copenhagen Business School, Denmark

1. Torkil Clemmensen, project coordinator of CultUsab

2. Lene Nielsen

3. Rikke Ørngreen

4. Qingxin Shi

Department of Design, Indian Institute of Technology Guwahati. Assam, India.

5. Pradeep Yammiyavar, project partner CultUsab

6. Jyoti Kumar

C-DAC, Pune, India

7. Dinesh Katre

Institute of Psychology, Chinese Academy of Sciences, Beijing, China

8. Xianghong Sun, project partner CultUsab

9. Huiyang $\mathrm{Li}$

10. Weina $\mathrm{Qu}$

11. Chenfu Cui

Dept. of Communication, Journalism and Computer Science, University of Roskilde

12. Morten Hertzum

Dept. of Computer Science at University of Copenhagen

13. Kasper Hornbæk

Snitker \& Co

14. Thomas Snitker

15. Steen Filtskov Andersen

16. Elisabeth Landbo

17. Troels Fibæk Bertel

18. Mimi Truong

Nokia Denmark

19. Niels Ebbe Jacobsen

Honeywell USA

20. Thomas Plocher

HFI Mumbai, India

21. Apala Chavan

22. Sachin Patil

23. Hemal Gathani 
Baidu, Beijing, China

24. Han Qi

Motorola Beijing, China

25. Yanfang Liu

26. Jing $\mathrm{Li}$

User Experience Technology Consulting Ltd. Beijing, China

27. Yiner Ya

28. Xiuling Lu

\section{Siemens Beijing, China}

29. Yifei $\mathrm{Xu}$

30. Xiangang Qin 